\title{
A Generic Nonlinear Aerodynamic Model for Aircraft
}

\author{
Jared A. Grauer* and Eugene A. Morelli ${ }^{\dagger}$ \\ NASA Langley Research Center, Hampton, Virginia, 23681
}

\begin{abstract}
A generic model of the aerodynamic coefficients was developed using wind tunnel databases for eight different aircraft and multivariate orthogonal functions. For each database and each coefficient, models were determined using polynomials expanded about the state and control variables, and an othgonalization procedure. A predicted squarederror criterion was used to automatically select the model terms. Modeling terms picked in at least half of the analyses, which totalled 45 terms, were retained to form the generic nonlinear aerodynamic (GNA) model. Least squares was then used to estimate the model parameters and associated uncertainty that best fit the GNA model to each database. Nonlinear flight simulations were used to demonstrate that the GNA model produces accurate trim solutions, local behavior (modal frequencies and damping ratios), and global dynamic behavior ( $91 \%$ accurate state histories and $80 \%$ accurate aerodynamic coefficient histories) under large-amplitude excitation. This compact aerodynamics model can be used to decrease on-board memory storage requirements, quickly change conceptual aircraft models, provide smooth analytical functions for control and optimization applications, and facilitate real-time parametric system identification.
\end{abstract}

\section{Nomenclature}

\begin{tabular}{|c|c|c|c|}
\hline \multicolumn{2}{|l|}{ Roman } & $V$ & airspeed $[\mathrm{ft} / \mathrm{s}]$ \\
\hline a & orthogonal model parameters & $\mathbf{X}$ & regressors \\
\hline$a_{x}, a_{y}, a_{z}$ & body accelerations $[\mathrm{G}]$ & $X, Y, Z$ & aerodynamic forces $[\mathrm{lbf}]$ \\
\hline & wing span $[\mathrm{ft}]$ & $x_{c g}$ & center of gravity position $[\mathrm{ft}]$ \\
\hline$C_{D}, C_{Y}, C_{L}$ & aerodynamic force coefficients & $\mathbf{y}$ & model output \\
\hline$C_{l}, C_{m}, C_{n}$ & aerodynamic moment coefficients & $\mathbf{z}$ & measurement \\
\hline $\begin{array}{l}C_{X}, C_{Z} \\
\operatorname{cov}(.)\end{array}$ & $\begin{array}{l}\text { longitudinal and heave coefficients } \\
\text { covariance }\end{array}$ & Greek & \\
\hline $\bar{c}$ & mean aerodynamic chord $[\mathrm{ft}]$ & $\alpha$ & angle of attack [rad] \\
\hline I & inertia tensor $\left[\right.$ slug. $\left.\mathrm{ft}^{2}\right]$ & $\beta$ & sideslip angle [rad] \\
\hline$J(\boldsymbol{\theta})$ & least-squares cost function & $\delta_{a}, \delta_{e}, \delta_{r}$ & aileron, elevator, rudder deflection [rad] \\
\hline$L, M, N$ & aerodynamic moments $[\mathrm{ft} \cdot \mathrm{lbf}]$ & $\theta$ & model parameters \\
\hline$m$ & mass [slug] & $\nu$ & measurement noise \\
\hline$N$ & number of observations & $\Xi$ & candidate regressors \\
\hline$n$ & number of parameters & $\sigma^{2}$ & covariance \\
\hline $\mathbf{P}$ & orthogonal regressors & $v$ & residual \\
\hline PSE & predicted squared error & & \\
\hline$p, q, r$ & roll, pitch, and yaw rates $[\mathrm{rad} / \mathrm{s}]$ & Superscripts & \\
\hline $\bar{q}$ & dynamic pressure $\left[\mathrm{lbf} / \mathrm{ft}^{2}\right]$ & & time derivative \\
\hline $\mathcal{R}$ & correlation matrix & $T$ & matrix transpose \\
\hline$R^{2}$ & coefficient of determination & $\sim$ & normalized rates $[\mathrm{rad}]$ \\
\hline$S$ & wing reference area $\left[\mathrm{ft}^{2}\right]$ & . & estimated value \\
\hline$T$ & thrust [lbf] & & \\
\hline$t$ & time $[\mathrm{s}]$ & & \\
\hline
\end{tabular}

\footnotetext{
*Research Engineer, Dynamic Systems and Control Branch, MS 308, Member AIAA

$\dagger$ Research Engineer, Dynamic Systems and Control Branch, MS 308, Member AIAA
} 


\section{Introduction}

Clobally valid, high-fidelity aerodynamic models used in applications such as flight simulation and $G$ feedback control design often come from wind tunnel test data in the form of databases of aerodynamic coefficients, tabulated according to state and control variables. There are several disadvantages to modeling aircraft aerodynamics in this manner. These databases are relatively large and encompass wide ranges of the flight envelope with sufficient resolution to accurately model the global, nonlinear aerodynamics of rigid-body aircraft. Large amounts of time, money, computational resources, and manpower are required to produce these databases. Various sources of error can make the aerodynamic coefficient hyper-surfaces appear ragged and discontinuous. This leads to problems when computing gradients for optimization, control analysis, trimming, and generating linear models. There is also no clear approach to smoothly update regions of the tables with new information from subsequent wind tunnel tests, computational fluid dynamics results, or flight tests. The tabular nature of these databases also makes it difficult to gain physical insight into the behavior of the aircraft by simple inspection.

Analytical models using functional expansions are often used to approximate the databases and address those problems. However, it is not always clear which and how many terms should be included in the models. Many works have used proper orthogonal decompositions, ${ }^{1}$ singular value decompositions, ${ }^{2,3,4,5,6}$ or Chebyshev polynomials ${ }^{7}$ to generate basis functions that approximate the databases. These methods however use many terms and the basis functions do not provide any physical insight into the aircraft dynamics. Step-wise regression is often used to generate model structures, however this method is iterative and is time consuming. Model terms can also be highly correlated, which causes inaccurate parameter estimates.

A relatively new technique that mitigates these problems is modeling aerodynamic data using multivariate orthogonal functions (MOFs). A large pool of terms based on aircraft states and controls are transformed into a set of orthogonal polynomials. The functions can be ordered in terms of importance in fitting the data because they are orthogonal. A statistical metric is then used to select the number of model terms to attain good accuracy without overparameterzing the model, which could increase uncertainty and decrease predictive capability. Ordinary least-squares parameter estimation is used to identify model parameters. This method has been successfully applied in several applications using flight-test data. ${ }^{8,9,10}$

This modeling method currently must be applied to each aircraft to determine the appropriate model structure and parameter estimates. However, conventional aircraft tend to behave similarly and it is expected that a large number of aircraft can be modeled reasonably well using the same aerodynamic model structure and only changing a few model parameters. Different types of aircraft could then quickly be changed for analysis. This would be advantageous for flight simulators, conceptual designs, and control law verification on different aircraft or using parametric uncertainties. The models are nonlinear and valid within large regions of the flight envelope. The analytical model requires only small amounts of memory and can produce smooth and differentiable data. With a known model structure, design of experiments can be used to lower wind tunnel test time and costs. Parametric system identification methods such as equation error in the frequency domain ${ }^{11,12,13}$ can also be used given a known model structure.

In this paper, a generic nonlinear aerodynamic (GNA) model is presented. Multivariate orthogonal functions were used to generate models of the aerodynamic coefficients, approximating aerodynamic databases generated from wind tunnel databases for eight different nonlinear flight simulations. Terms that were identified in at least half of the analyses are retained in the generic model. Ordinary least-squares parameter estimation was then used with that model structure and the original aerodynamic databases to estimate the model parameters for each aircraft. Substitution of these identified generic aerodynamic models back into the flight simulations and excitation using dynamic maneuvers show that these models approximate the databases well.

This paper is organized as follows. Section II presents the aerodynamic coefficients, ordinary least squares, and model structure determination using multivariate orthogonal functions. Section III describes the eight nonlinear flight simulations used to identify models. Section IV presents the generic aerodynamic model structure, model parameters for the nonlinear simulations, and comparisons with the original aerodynamic databases using dynamic maneuvers.

Computer programs for modeling with multivariate orthogonal functions, least-squares regression with colored residuals, and the F-16C nonlinear simulation are all included in a MATLAB ${ }^{\circledR}$ toolbox called System

IDentification Programs for AirCraft (SIDPAC). ${ }^{14}$ This software was developed at NASA Langley Research Center and is continually expanded and improved upon. 


\section{Methods}

\section{II.A. Aerodynamic Coefficients}

Models were developed for the aerodynamic force and moment coefficients. These were computed from the body-frame applied forces $X, Y, Z$ and moments $L, M, N$ as

$$
\begin{gathered}
{\left[\begin{array}{c}
C_{D} \\
C_{Y} \\
C_{L}
\end{array}\right]=\frac{1}{\bar{q} S}\left[\begin{array}{ccc}
-\cos \alpha & 0 & -\sin \alpha \\
0 & 1 & 0 \\
+\sin \alpha & 0 & +\cos \alpha
\end{array}\right]\left[\begin{array}{c}
X \\
Y \\
Z
\end{array}\right]} \\
{\left[\begin{array}{c}
C_{l} \\
C_{m} \\
C_{n}
\end{array}\right]=\frac{1}{\bar{q} S}\left[\begin{array}{ccc}
1 / b & 0 & 0 \\
0 & 1 / \bar{c} & 0 \\
0 & 0 & 1 / b
\end{array}\right]\left[\begin{array}{c}
L \\
M \\
N
\end{array}\right]}
\end{gathered}
$$

where $\alpha$ is the angle of attack, $\bar{q}$ is the dynamic pressure, $S$ is the wing reference area, $\bar{c}$ is the mean aerodynamic chord, and $b$ is the wing span. Drag and lift forces were used instead of body-frame longitudinal and heave forces because the aerodynamics are natively written in the wind frame, which results in simpler models. This form is appropriate when measuring forces and moments, for example during a wind tunnel test. If instead flight test data are available, standard modeling assumptions can be used ${ }^{15,16,14}$ to compute these as

$$
\begin{gathered}
{\left[\begin{array}{c}
C_{D} \\
C_{Y} \\
C_{L}
\end{array}\right]=\frac{1}{\bar{q} S}\left[\begin{array}{ccc}
-\cos \alpha & 0 & -\sin \alpha \\
0 & 1 & 0 \\
+\sin \alpha & 0 & +\cos \alpha
\end{array}\right]\left[\begin{array}{c}
m a_{x}-T \\
m a_{y} \\
m a_{z}
\end{array}\right]} \\
{\left[\begin{array}{c}
C_{l} \\
C_{m} \\
C_{n}
\end{array}\right]=\frac{1}{\bar{q} S}\left[\begin{array}{ccc}
1 / b & 0 & 0 \\
0 & 1 / \bar{c} & 0 \\
0 & 0 & 1 / b
\end{array}\right]\left[\begin{array}{c}
I_{x x} \dot{p}-I_{x z}(p r+\dot{r})+\left(I_{x z}-I_{y y}\right) q r \\
I_{y y} \dot{q}+\left(I_{x x}-I_{z z}\right) p r+I_{x z}\left(p^{2}-r^{2}\right) \\
I_{z z} \dot{r}-I_{x z}(\dot{p}-q r)+\left(I_{y y}-I_{x x}\right) p q
\end{array}\right]}
\end{gathered}
$$

where $T$ is the engine thrust, $a_{x}, a_{y}, a_{z}$ are linear accelerations, $p, q, r$ are the body rates, $m$ is the mass, and $\left\{I_{i j}\right\}$ are elements of the inertia tensor.

\section{II.B. Ordinary Least Squares}

Consider the model

$$
\begin{aligned}
\mathbf{z} & =\mathbf{y}+\boldsymbol{\nu} \\
& =\mathbf{X} \boldsymbol{\theta}+\boldsymbol{\nu}
\end{aligned}
$$

for $N$ measurements of $\mathbf{z}$, where $\mathbf{y}$ is the model output, $\mathbf{X}=\left[\begin{array}{llll}\mathbf{x}_{1} & \mathbf{x}_{2} & \ldots & \mathbf{x}_{n}\end{array}\right]$ is a matrix of $n$ independent regressor variables, $\boldsymbol{\theta}$ is a vector of model parameters, and $\boldsymbol{\nu}$ is the measurement error. The least-squares cost function

$$
J(\boldsymbol{\theta})=\frac{1}{2}(\mathbf{z}-\mathbf{X} \boldsymbol{\theta})^{T}(\mathbf{z}-\mathbf{X} \boldsymbol{\theta})
$$

is minimized by the solution

$$
\hat{\boldsymbol{\theta}}=\left(\mathbf{X}^{T} \mathbf{X}\right)^{-1} \mathbf{X}^{T} \mathbf{z}
$$

The uncertainties of the estimated parameters are

$$
\operatorname{cov}(\hat{\boldsymbol{\theta}})=\left(\mathbf{X}^{T} \mathbf{X}\right)^{-1}\left[\sum_{i=1}^{N} \mathbf{x}(i) \sum_{j=1}^{N} \mathcal{R}_{\nu \nu}(i-j) \mathbf{x}^{T}(j)\right]\left(\mathbf{X}^{T} \mathbf{X}\right)^{-1}
$$

where $\mathcal{R}_{\nu \nu}$ is the autocorrelation of the residuals. This can be estimated as

$$
\hat{\mathcal{R}}_{\nu \nu}(k)=\frac{1}{N} \sum_{i=1}^{N-k} \boldsymbol{v}(i) \boldsymbol{v}(i+k) \quad \text { for } k=0,1,2, \ldots, N-1
$$


where

$$
\boldsymbol{v}=\mathbf{z}-\mathbf{y}
$$

are the residuals. ${ }^{17,18,14}$ Equation (6) is necessary to accurately predict the uncertainties in this work because of deterministic content left in the residuals due to model truncation.

There are two important problems with using ordinary least squares to estimate a generic aerodynamic model for aircraft. The first is that in order to solve Eq. (5), the model structure must be known. Sometimes prior knowledge can be used, other times step-wise regression can determine model structures using a variety of statistical metrics. However, the model structure problem must be determined iteratively until a solution is deemed sufficient. Another problem is that regressors typically have some level of correlation, either from feedback control, small ranges in which variables are similar, or simply the motion of the aircraft. In these cases, the least-squares estimator cannot correctly attribute variation to the correct regressor and the $\mathbf{X}^{T} \mathbf{X}$ matrix becomes ill-conditioned. These problems make using ordinary least squares difficult for identifying and estimating generic aircraft models from data.

\section{II.C. Model Structure Determination using Multivariate Orthogonal Functions}

The problems with ordinary least squares mentioned in the last section can be overcome by using multivariate orthogonal functions. This method orthgonalizes the regressors so that their unique variations become apparent. In the process, model terms can be ordered by the amount in which they lower the cost. Using a statistical metric, the process can be automated to choose the most important terms that model the measurement well without overparameterizing the model. This process has been successfully applied to numerous research problems, documented in several references, ${ }^{8,9,14}$ and is briefly summarized here.

The process begins by selecting a matrix of $n$ candidate regressor variables $\boldsymbol{\Xi}=\left[\begin{array}{llll}\boldsymbol{\xi}_{1} & \boldsymbol{\xi}_{2} & \ldots & \boldsymbol{\xi}_{n}\end{array}\right]$ to be orthogonalized. The orthogonal modeling functions are

$$
\begin{aligned}
& \mathbf{p}_{0}=\mathbf{1} \\
& \mathbf{p}_{j}=\boldsymbol{\xi}_{j}-\sum_{k=0}^{j-1} \gamma_{k j} \mathbf{p}_{k} \quad j=1,2, \ldots, n
\end{aligned}
$$

where for convenience the first function is selected as unity and then the remaining functions are defined recursively. During this process, the coefficients

$$
\gamma_{k j}=\frac{\mathbf{p}_{k}^{T} \boldsymbol{\xi}_{j}}{\mathbf{p}_{k}^{T} \mathbf{p}_{k}} \quad k=0,1, \ldots, j-1
$$

are defined, which populate the upper-triangular matrix

$$
\mathbf{G}=\left[\begin{array}{ccccc}
1 & \gamma_{01} & \gamma_{02} & \ldots & \gamma_{0 n} \\
0 & 1 & \gamma_{12} & \ldots & \gamma_{1 n} \\
0 & 0 & 1 & \ldots & \gamma_{2 n} \\
\vdots & \vdots & \vdots & \ddots & \vdots \\
0 & 0 & 0 & \ldots & 1
\end{array}\right]
$$

The matrix $\mathbf{G}$ is a linear transform that orthgonalizes the candidate regressor variables as

$$
\mathbf{P}=\left[\begin{array}{llll}
\mathbf{p}_{0} & \mathbf{p}_{1} & \ldots & \mathbf{p}_{n}
\end{array}\right]=\mathbf{\Xi} \mathbf{G}^{-1}
$$

where $\mathbf{p}_{i}^{T} \mathbf{p}_{j}=0$ for $i \neq j$. The matrix $\mathbf{P}^{T} \mathbf{P}$ is diagonal and by applying Eq. (5), the orthgonal parameter estimates are

$$
\hat{a}_{j}=\left(\mathbf{p}_{j}^{T} \mathbf{z}\right) /\left(\mathbf{p}_{j}^{T} \mathbf{p}_{j}\right)
$$

which depend only on the data and the regressor, and does not depend on the other model terms. Substituting Eq. (13) back into Eq. (4), the least-squares cost function is ${ }^{8,14}$

$$
J(\hat{\mathbf{a}})=\frac{1}{2} \mathbf{z}^{T} \mathbf{z}-\frac{1}{2} \sum_{j=0}^{n}\left(\mathbf{p}_{j}^{T} \mathbf{z}\right)^{2} /\left(\mathbf{p}_{j}^{T} \mathbf{p}\right)
$$


where the summation term is the value by which including that model parameter in the model reduces the cost. By ordering the regressors in terms of this value, they are ordered in terms of their effectiveness in modeling the data. Orthogonalizing the regressors has decoupled least-squares in that each model parameter can be independently estimated and judged on how effective it is in reducing the cost function.

A metric called the predicted squared error

$$
\mathrm{PSE}=\frac{1}{N}(\mathbf{z}-\hat{\mathbf{y}})^{T}(\mathbf{z}-\hat{\mathbf{y}})+\sigma_{\max }^{2} \frac{n}{N}
$$

can be employed to determine how many regressors should be retained in the final model. ${ }^{19,8,14}$ The first term is called the mean squared fit error (MSFE), which monotonically decreases with each additional modeling term and penalizes the error in the model fit. The second term is the over-fit penalty (OFP), which monotonically increases and guards against over-parameterizing the model, which leads to poor prediction results. Since one term is always increasing and one is always decreasing, there is always a point at which the PSE is minimized. Choosing this point for selecting the number of modeling terms results in good models. The variance $\sigma_{\max }^{2}$ is of the mean measurements, which is conservative in that it produces models with minimal complexity. Once the minimum PSE is obtained, the final regressor variable matrix $\mathbf{X}$ can be assembled, and ordinary least squares can be used as normal. This procedure is automated within a code contained in SIDPAC. ${ }^{14}$

\section{Aircraft Nonlinear Flight Simulations}

Eight nonlinear simulations of different aircraft flight dynamics models were used. These aircraft simulations and data are documented in several references. ${ }^{20,14,21}$ The aerodynamic data for these models comes from static and forced-oscillation tests conducted at various wind tunnels at NASA Langley Research Center.

The aircraft used were the A-7, F-4, F-16C, F-16XL, F-106B, Generic Transport Model (GTM), Twin Otter (TO), and X-31. The A-7 is a single-seat attack fighter. The F-4 is a two-seat fighter/bomber aircraft. The F-16C is a single-seat, multi-role fighter aircraft. The F-16XL is an enhanced version of the F-16C with a cranked-arrow delta wing configuration. The F-106B is a two-seat interceptor with a delta wing configuration. The GTM is a sub-scale model of a typical transport-style aircraft. The TO is a commuter style research aircraft. The X-31 is a single-seat research aircraft with a cranked delta wing configuration and thrust-vectoring capability, used for research in highly agile flight. The majority of these aircraft are agile aircraft capable of seating one or two pilots. There are two larger style transport aircraft.

The aerodynamic databases and nonlinear aircraft simulations were previously coded in MATLAB ${ }^{\circledR} .20,14,21$ Table 1 lists the mass and geometry properties for each of these aircraft. The longitudinal position of the aircraft center of gravity $x_{c g}$ has been changed in some instances to make the simulations flyable without feedback control. The simulations include routines for trimming the aircraft, generating linear models from numerical finite-differences, computing aerodynamic coefficients from the states and surface deflections, and simulating the dynamic response of the aircraft to control inputs.

\section{Results}

This section presents the generic aerodynamic model, determined using the aforementioned methodology. All aircraft simulations described in Section III were used in the analysis. For illustration, the GTM results are highlighted because this is a well-known and high-fidelity simulation that is available to the public.

\section{IV.A. Model Structure Determination}

The aerodynamic databases were interrogated to obtain measurements of the aerodynamic coefficients at different conditions. For computational tractability, this interrogation was performed separately for the longitudinal and lateral/direction dynamics because typically these are only weakly coupled in normal flight regimes. A total of 3168 longitudinal and 9072 lateral/directional cases were analyzed, using the ranges and resolutions of independent variables listed in Table 2. The ranges selected remain within those used during the wind tunnel testing used to develop the simulations, and also remain within the normal operating envelope. ${ }^{22}$ The resolutions selected are similar to what was used in the wind tunnel testing to refrain from artificially lowering the uncertainty on the estimation results. To lower the number of database interrogations, 
it was also assumed that the aircraft have lateral symmetry, for instance so that an aileron deflection in each direction causes the same magnitude roll rate. During the longitudinal interrogation, all lateral/directional states and deflections were set to null, and vice-versa.

For each aircraft, the procedure described in Section II was used to determine model structures for each aerodynamic coefficient in Eq. (1) and then to estimate model parameters and uncertainties for those models. Independent variables for the longitudinal interrogation were $\alpha, \tilde{q}$, and $\delta_{e}$, whereas $\beta, \tilde{p}, \tilde{r}, \delta_{a}$, and $\delta_{r}$ were used for the lateral/directional interrogations, where

$$
\left[\begin{array}{c}
\tilde{p} \\
\tilde{q} \\
\tilde{r}
\end{array}\right]=\frac{1}{2 V}\left[\begin{array}{ccc}
b & 0 & 0 \\
0 & \bar{c} & 0 \\
0 & 0 & b
\end{array}\right]\left[\begin{array}{l}
p \\
q \\
r
\end{array}\right]
$$

are the non-dimensional body rates, where $V$ is the airspeed. Variables were taken in combinations up to fourth order, e.g., $1, \alpha, \tilde{q}, \delta_{e}, \alpha \delta_{e}, \alpha^{2} \tilde{q} \delta_{e}, \alpha^{4}$, to form the candidate regressor pool. Mach effects were not considered because the interrogations were restricted to subsonic velocities $(M=0.1)$ where the variation in airspeed is removed using non-dimensional aerodynamic coefficients. Thrust effects were not considered since they contribute only second-order interactions with the aerodynamics. Any additional control surface deflections, such as flaps and canards, were set to null for this analysis.

Figure 1 shows the roll moment coefficient orthogonal function modeling results for the GTM. As more functions are included in the model, the MSFE decreases and the OFP increases. The PSE is minimized when the first ten orthogonal functions are included. However only nine functions were included because upon further analysis, one of those functions was linearly dependent on another two. These functions, ordered in importance to the model, are

$$
\mathbf{X}=\left[\begin{array}{lllllllll}
1 & \beta & \delta_{r} & \tilde{p} & \delta_{a} & \beta^{3} & \tilde{r} & \delta_{r}^{2} & \beta \delta_{a}
\end{array}\right]
$$

which had $R^{2}=0.9997$ and $\sigma_{v}=0.0003$, indicating a good fit. The fit for this model and the residuals are shown in Figure 2. Data is shown for each interrogation because the roll coefficient is a multiple-dimension hyper-surface. The plot points out the magnitude of the roll coefficient, shows the model fits the data very closely, and illustrates the residuals are small.

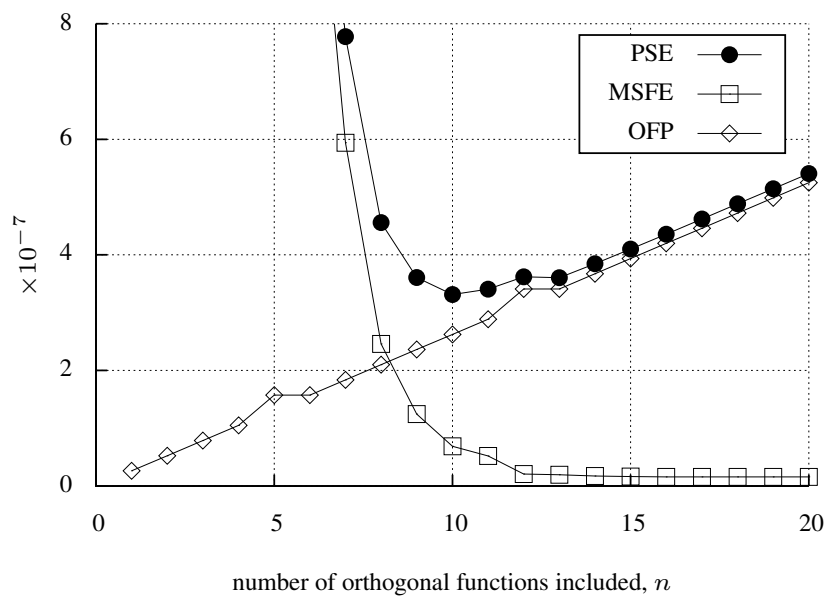

Figure 1. GTM roll coefficient modeling using multivariate orthogonal functions

Application of multivariate orthogonal function modeling resulted in different aerodynamic models for each aircraft. Modeling terms that were used in at least half of the aircraft models were retained in the GNA model. For instance, Figure 3 displays all the important modeling terms for the roll coefficient, as well as the number of instances in which they were selected. The roll rate and aileron deflection were selected for each of the eight aircraft, which is not surprising since these two variables comprise the firstorder roll mode approximation. ${ }^{15,16,14}$ The sideslip angle, yaw rate, and rudder deflection were selected for seven out of the eight aircraft, which again is not surprising because these variables are coupled in the linearized lateral/directional modes of conventional aircraft. The remainder of the modeling terms are 

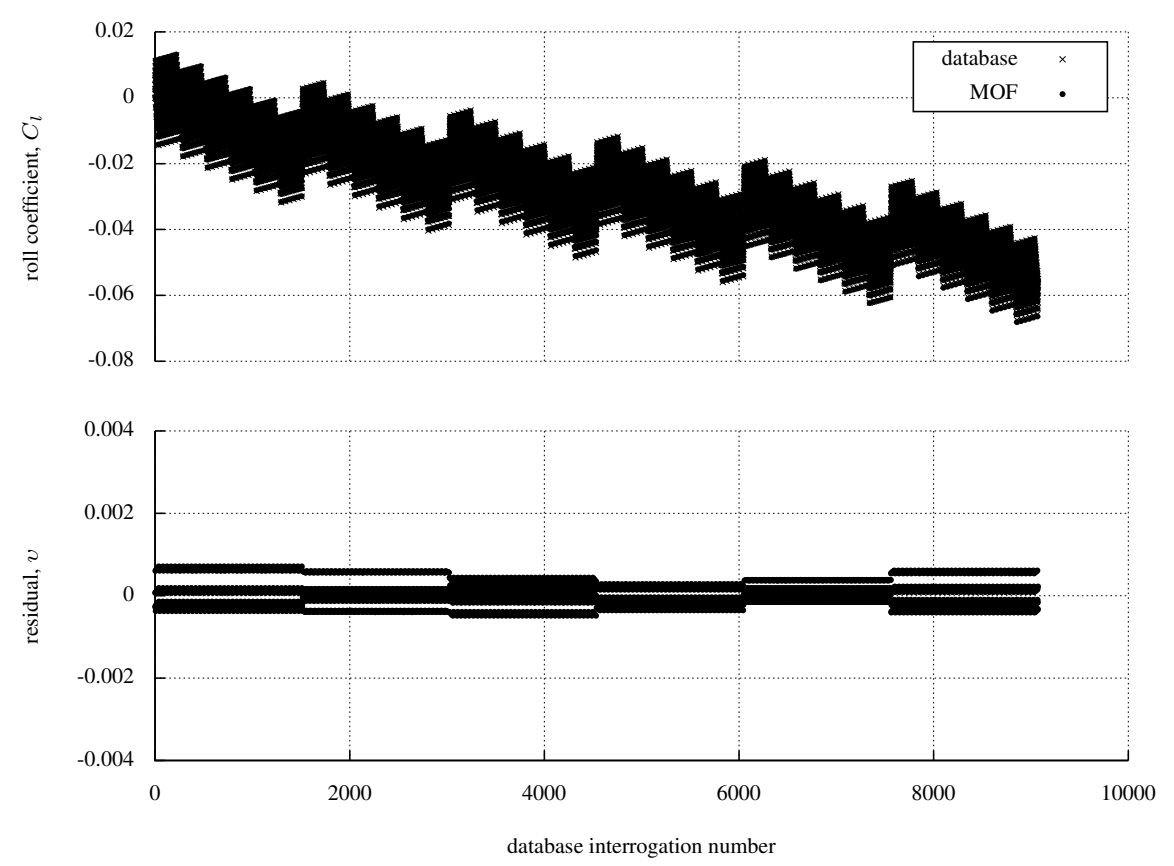

Figure 2. GTM roll coefficient database interrogation and fitting using multivariate orthogonal functions

nonlinear variables that appear in less than half of the aircraft models. This disparity supports the decision to use the majority, i.e. at least four of the eight, as a good cut-off point for selecting which modeling terms are retained in the general nonlinear aerodynamic model.

This process was applied to all of the aerodynamic coefficients, and the final structure for the GNA model is

$$
\begin{aligned}
C_{D} & =\theta_{1}+\theta_{2} \alpha+\theta_{3} \alpha \tilde{q}+\theta_{4} \alpha \delta_{e}+\theta_{5} \alpha^{2}+\theta_{6} \alpha^{2} \tilde{q}+\theta_{7} \alpha^{2} \delta_{e}+\theta_{8} \alpha^{3}+\theta_{9} \alpha^{3} \tilde{q}+\theta_{10} \alpha^{4} \\
C_{Y} & =\theta_{11} \beta+\theta_{12} \tilde{p}+\theta_{13} \tilde{r}+\theta_{14} \delta_{a}+\theta_{15} \delta_{r} \\
C_{L} & =\theta_{16}+\theta_{17} \alpha+\theta_{18} \tilde{q}+\theta_{19} \delta_{e}+\theta_{20} \alpha \tilde{q}+\theta_{21} \alpha^{2}+\theta_{22} \alpha^{3}+\theta_{23} \alpha^{4} \\
C_{l} & =\theta_{24} \beta+\theta_{25} \tilde{p}+\theta_{26} \tilde{r}+\theta_{27} \delta_{a}+\theta_{28} \delta_{r} \\
C_{m} & =\theta_{29}+\theta_{30} \alpha+\theta_{31} \tilde{q}+\theta_{32} \delta_{e}+\theta_{33} \alpha \tilde{q}+\theta_{34} \alpha^{2} \tilde{q}+\theta_{35} \alpha^{2} \delta_{e}+\theta_{36} \alpha^{3} \tilde{q}+\theta_{37} \alpha^{3} \delta_{e}+\theta_{38} \alpha^{4} \\
C_{n} & =\theta_{39} \beta+\theta_{40} \tilde{p}+\theta_{41} \tilde{r}+\theta_{42} \delta_{a}+\theta_{43} \delta_{r}+\theta_{44} \beta^{2}+\theta_{45} \beta^{3}
\end{aligned}
$$

which uses 45 model terms. With the exception of the drag coefficient, all the linear terms are present in the aerodynamic coefficients. The sideforce and roll coefficients are strictly linear. The drag, lift, and pitching moment coefficients are the most nonlinear, having terms ranging up to $\alpha^{4}$ to model variations due to stall. The yaw coefficient is the only lateral/directional coefficient having nonlinear terms, where the $\beta^{3}$ term is used to model the asymmetric variations at higher sideslip.

\section{IV.B. Parameter Estimation}

Ordinary least-squares parameter estimation described in Section II was used to estimate the model parameters that best fit the GNA model in Eq. (17) to the aerodynamic database for each aircraft. Parameter estimates and standard errors are reported for each aircraft in Tables 3 and 4 . Together with the mass and geometry properties provided in Table 1, this information can be used to build nonlinear flight simulations of each of the aircraft in this paper.

When comparing the multivariate orthogonal function model to the GNA model, there are four possible cases. The first case is that the models contain the same modeling terms, which results in the models being the same. The second case is that the GNA model contains more terms than the MOF model. The GNA model is overparameterized in this case, which results in variations of the parameter estimates, larger uncertainties, and small values for the extra parameters. The third case is when the GNA model contains fewer terms than the MOF model. In this case variations in the database are not captured by the GNA 


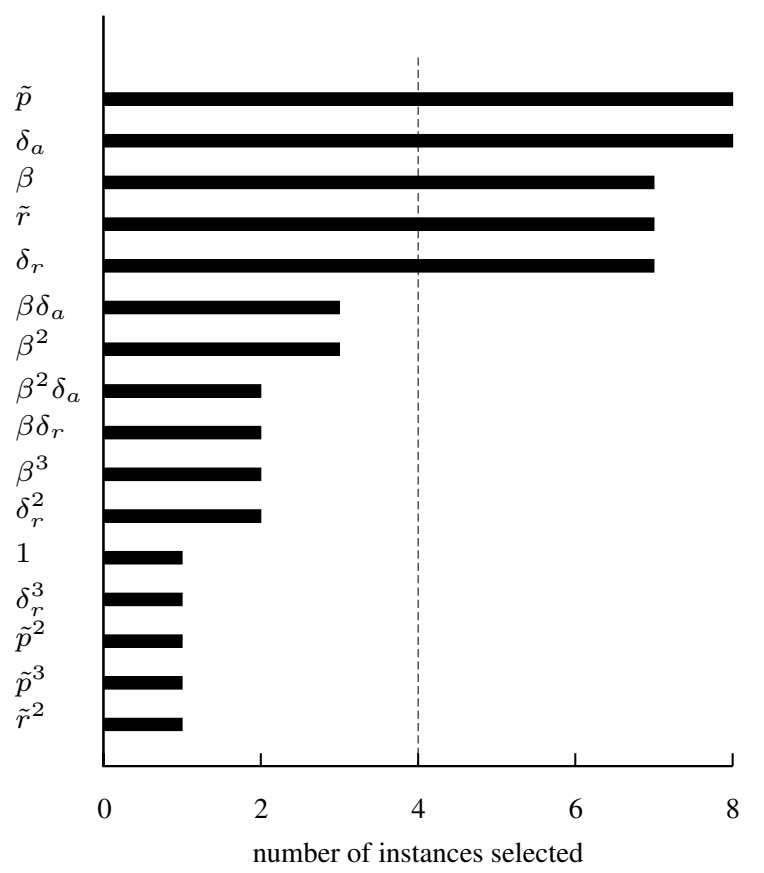

Figure 3. Modeling terms and instances for the roll coefficient model using aircraft multivariate orthogonal functions

model, which results in poorer fits to the data and larger error bounds. The fourth case is when different variables are used to model the variation. While this shifts the modeling dependencies, it does not necessarily lower the fit or increase the error on estimated parameters.

In general, the GNA model fit the aircraft aerodynamic databases well. The fits had ranges of $R^{2}$ between 0.8676 and 1.0000 , indicating good fits. For example, Figure 4 shows the fitting of the GNA model to the database for the GTM roll coefficient. The GNA model has $R^{2}=0.9961$ and $\sigma_{v}=0.0010$, which fit the data well. Compared with Figure 2, the residual is larger for the GNA model, which is due to the difference in the model structures. This highlights one of the fundamental compromises with using reduced-order models, that model simplicity is gained at the expense of model accuracy.

This GNA model can also be used for preliminary design and for testing control laws for a broad range of aircraft. For instance, Figure 5 shows the parameter estimates and two standard deviation error bounds of the model parameter $\theta_{30}$, which is the traditional pitching moment stiffness derivative $C_{m_{\alpha}}$. All values lie within the typical range $-3 \mathrm{rad}$ to $+1 \mathrm{rad},{ }^{23}$ and are consistent with results for previous aircraft. ${ }^{24,16}$ The fighters have lower values because they are designed with lower static margins than the GTM and Twin Otter, which are transport and commuter style aircraft. The error bounds on the GTM and Twin Otter estimates are higher than the other aircraft because the MOF analysis wanted to use more and other parameters than those retained in the GNA model, which led to larger residuals and error bounds. For an agile fighter type aircraft, select a value of $C_{m_{\alpha}}$ around -0.4 , for a transport-type aircraft, pick a value around -1.6 for conceptual design or testing. While these pitching moment stiffness terms are almost exactly equal to those found by linearizing the models numerically, it should be noted that the longitudinal position of the centers of mass have been artificially moved in the nonlinear simulations, per Table 1, so that normally unstable aircraft can be flown without control laws by a pilot in the simulation.

\section{IV.C. Validation}

To validate the GNA models, they were substituted for the original aerodynamic databases in the nonlinear flight simulations. The simulations were then excited using standard inputs for system identification. It was a very quick procedure to replace large database files with six equations. The simulations were trimmed and then large-amplitude doublets were applied to the elevator, aileron, and rudder control surfaces. Computerized inputs were used instead of piloted inputs to help excite nonlinear responses.

Figure 6 shows the results for the GTM simulation. The time histories of the control inputs are shown in Figure 6(a). The GTM was originally trimmed for straight and level flight at $1200 \mathrm{ft}$ with a $125 \mathrm{ft} / \mathrm{s}$ 

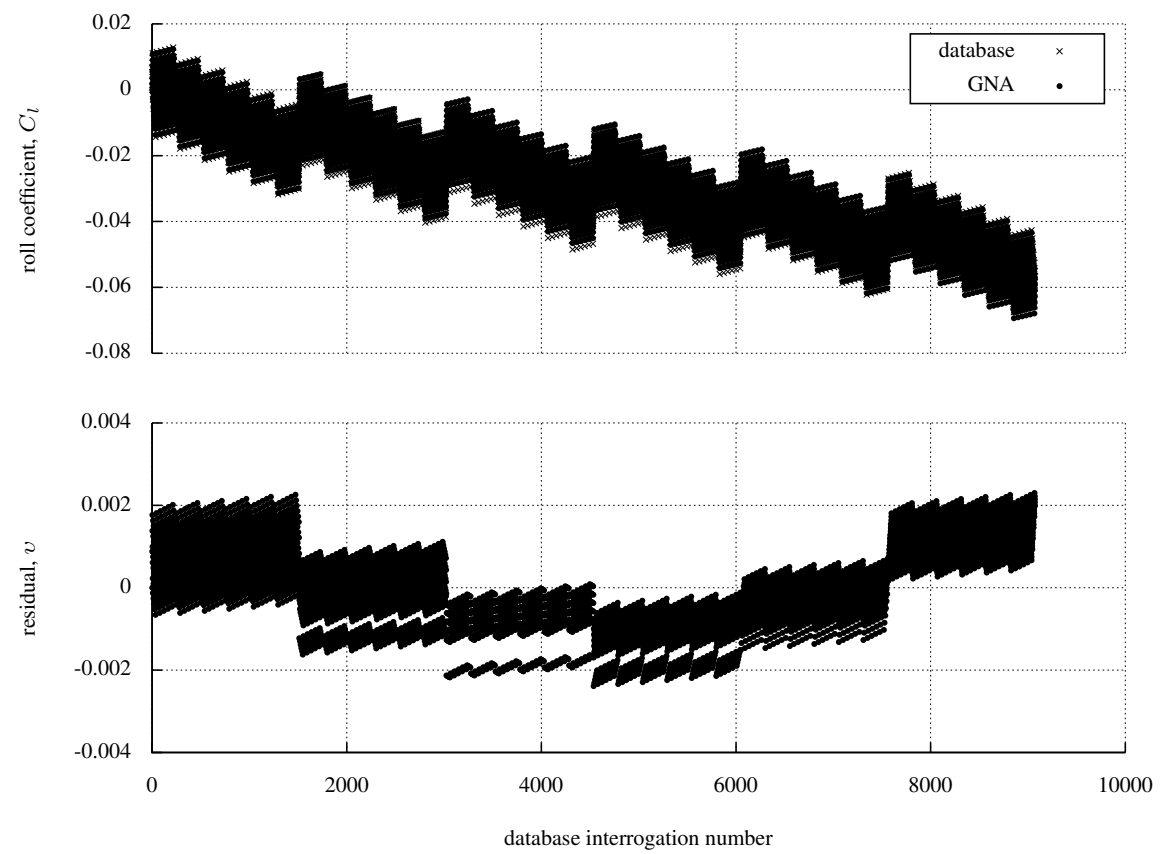

Figure 4. GTM roll coefficient database interrogation and fitting using generic nonlinear aerodynamic model

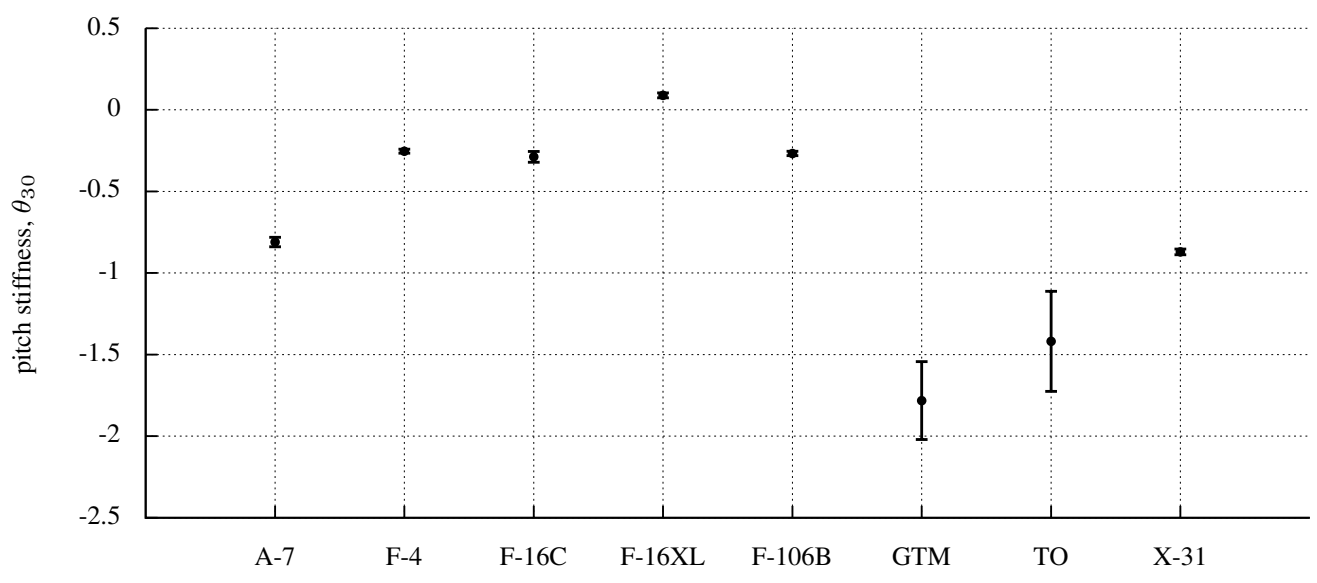

Figure 5. Identified model term for pitching moment stiffness $\theta_{30}$ and two standard deviation error bounds 
airspeed and 5.0 degree angle of attack using $15 \%$ throttle and 1.0 degree of elevator deflection. Using the GNA model, the throttle and elevator trim settings changed to $12 \%$ and 1.8 degrees, respectively. These trim conditions are very close, as seen in the starting values in Figure 6(a).

The time histories in Figure 6(a) are very close and had fits above $R^{2}=0.9094$. Differences between the time histories are attributed to using relatively small and low-order polynomial functions to approximate the large aerodynamic database. Figure 6(b) shows the time histories of the aerodynamic coefficients, using mass and geometry data in Table 1, Eq. (2), and the data in Figure 6(a). Again these time histories are very similar, with fits above $R^{2}=0.8008$, where the differences attributed to the differences in the aerodynamic models. The global, nonlinear dynamic behavior is very close between the original wind tunnel database and the compact GNA model.

Table 5 shows the modal parameters for the GTM about these trim conditions using both aerodynamic sources, obtained using numerical central finite-difference approximations. The same modes are present and the values reflect the approximate same modal behavior. The small differences in the results indicate that the GNA model is an excellent approximation of the aerodynamic databases. Local linear models, handling qualities, and modal parameters are very close between the database and GNA model.
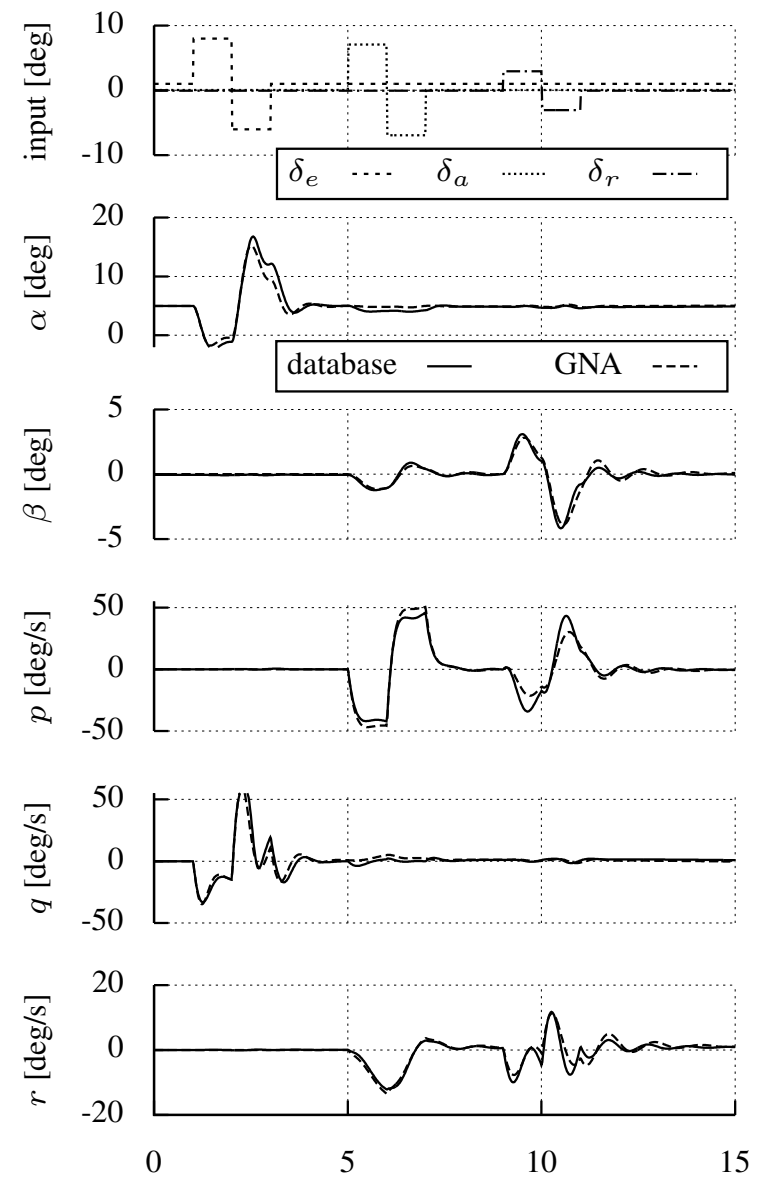

time, $t[\mathrm{~s}]$

(a) independent variable perturbation time histories
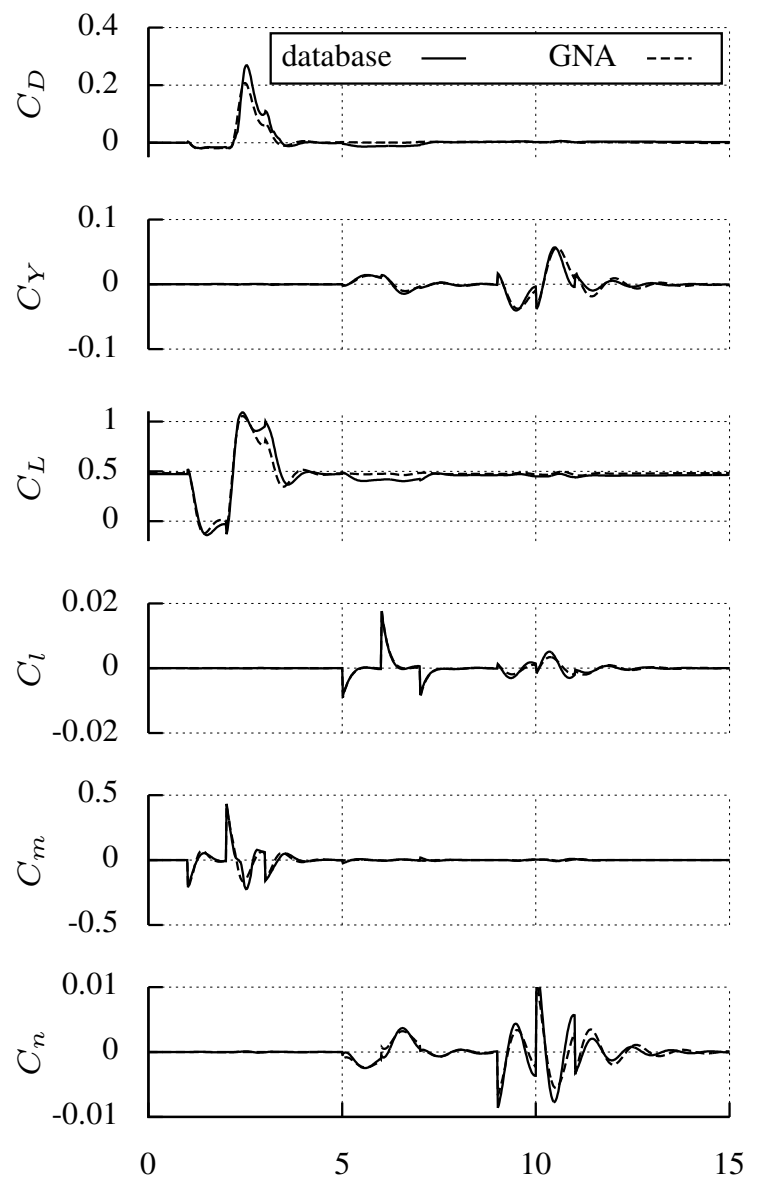

time, $t[\mathrm{~s}]$

(b) aerodynamic coefficient perturbation time histories

Figure 6. GTM independent modeling variable time histories under sequential doublet excitation 


\section{Conclusions}

This paper presented a generic nonlinear aerodynamics model for aircraft. This was accomplished by interrogating measured aerodynamic databases for eight aircraft over a large range of aerodynamic angles, body rates, and control surface deflections. This data was then used with multivariate orthogonal function modeling to determine nonlinear polynomial models for the aerodynamic coefficients. By minimizing the predicted square error, these models are both accurate and simple. The GNA model structure are the model terms deemed important in at least half of the analyses. Ordinary least squares was used to identify model parameters that best match the GNA model structure to the interrogated database, and these were substituted into nonlinear simulations of the aircraft to validate their accuracy. Values necessary for building nonlinear flight dynamic simulations for all eight aircraft presented are contained within this paper.

A single, fixed aerodynamic model structure could accurately approximate large aerodynamic databases for eight different aircraft, including fighter, fighter/bomber, research, commuter, and transport styles. It was demonstrated using the GTM that by using this method, trim solutions are accurately computed, local modal behavior is preserved, and $91 \%$ and $80 \%$ accuracy of large-amplitude state and aerodynamic coefficient time histories are obtained.

Having a GNA model makes it very easy to perform analyses on different types of aircraft. Simulations need only to switch 45 variables instead of large databases of aerodynamic measurements. Conceptual designers can change a few parameters according to historical trends, rules of thumb, or first principles to obtain dynamic flight simulations of new aircraft. Control law designers can change parameters to check performance for a large range of aircraft. Having functional representations of the aerodynamics allows for analytical derivation of derivatives for optimization applications. Having a known model structure facilitates real-time parameter estimation techniques.

This work could potentially be improved by incorporating more aircraft, different configurations, and larger ranges of the flight envelope into the analysis. If higher speeds are of interest, Mach effects could be included in the pool of candidate regressors. Assumptions of longitudinal and lateral/directional decoupling could be relaxed if more computational resources are available. In that case, it could be that some cross variables, such as $\alpha \beta$, could model variations that are currently attributed to higher order nonlinear functions. This work could also be extended to account for Mach variations, thrust and power effects, and variations due to additional control effectors. Other methods of selecting the GNA model terms are also possible.

\section{Acknowledgments}

This research funded by the NASA Aviation Safety Program, Vehicle Systems Safety Technologies project, and the Subsonic Fixed-Wing Project

\section{References}

${ }^{1}$ Dowell, E., "Eigenmode Analysis in Unsteady Aerodynamics: Reduced Order Models," No. AIAA-95-1450-CP in Structures, Structural Dynamics, and Materials Conference, AIAA/ASME/ASCE/AHS/ASC, New Orleans, LA, April 1995, pp. $2545-2557$.

${ }^{2}$ Lorente, L., Vega, J., and Velazquez, A., "Generation of Aerodynamic Databases Using High-Order Singular Value Decomposition," Journal of Aircraft, Vol. 45, No. 5, September-October 2008, pp. 1779-1788.

${ }^{3}$ Lorente, L., Vega, J., and Velazquez, A., "Compression of Aerodynamic Databases Using High-Order Singular Value Decomposition," Aerospace Science and Technology, Vol. 14, No. 3, May 2010.

${ }^{4}$ Castillo-Negrete, D., Hirshman, S., Spong, D., and D'Azevedo, E., "Compression of Magnetohydrodynamic Simulation Data Using Singular Value Decomposition," Journal of Computational Physics, Vol. 222, 2007, pp. 265-286.

${ }^{5}$ Miled, Z., Huian, L., Bukhres, O., Bem, M., Jones, R., and Oppelt, R., "Data Compression in a Pharmaceutical Drug Candidate Database," Informatica, Vol. 27, 2003, pp. 213-223.

${ }^{6}$ Kanth, K., Agrawal, D., Abbadi, A., and Singh, A., "Dimensionality Reduction for Similarity Searching in Dynamic Databases," Computational Vision Image Underst, Vol. 75, 1999, pp. 59-72.

${ }^{7}$ Leng, G., "Compression of Aircraft Aerodynamic Database Using Multivariate Chebyshev Polynomials," Advances in Engineering Software, Vol. 28, 1997, pp. 133-141.

${ }^{8}$ Morelli, E., "Global Nonlinear Aerodynamic Modeling Using Multivariate Orthogonal Functions," Journal of Aircraft, Vol. 32, No. 2, March-April 1995, pp. 270-277.

${ }^{9}$ Morelli, E., "Global Nonlinear Parametric Modeling with Application to F-16 Aerodynamics," No. i-98010-2, American Controls Conference, Philadelphia, PA, June 1998.

${ }^{10}$ Morelli, E., "Transfer Function Identification using Orthogonal Fourier Transform Modeling Functions," No. 2013-4749 in Atmospheric Flight Mechanics Conference, AIAA, Boston, MA, August 2013. 
${ }^{11}$ Morelli, E., "Real-Time Parameter Estimation in the Frequency Domain," Journal of Guidance, Control, and Dynamics, Vol. 23, No. 5, Sept.-Oct. 2000, pp. 812-818.

${ }^{12}$ Morelli, E., "Real-Time Dynamic Modeling: Data Information Requirements and Flight-Test Results," Journal of Aircraft, Vol. 46, No. 6, Nov.-Dec. 2009, pp. 1894-1905.

${ }^{13}$ Morelli, E., "Flight-Test Experiment Design for Characterizing Stability and Control of Hypersonic Vehicles," Journal of Guidance, Control, and Dynamics, Vol. 32, No. 3, May-June 2009, pp. 949-959.

${ }^{14}$ Klein, V. and Morelli, E., Aircraft System Identification: Theory and Practice, AIAA Education Series, AIAA, 2006.

${ }^{15}$ McRuer, D., Ashkenas, I., and Graham, D., Aircraft Dynamics and Automatic Control, Princeton, 1973.

${ }^{16}$ Stevens, B. and Lewis, F., Aircraft Control and Simulation, Wiley, 2nd ed., 2003.

${ }^{17}$ Morelli, E., "Determining the Accuracy of Maximum Likelihood Parameter Estimates with Colored Residuals," Tech. Rep. 194893, NASA, Hampton, VA, 1994.

${ }^{18}$ Morelli, E. and Klein, V., "Accuracy of Aerodynamic Model Parameters Estimated from Flight Test Data," Journal of Guidance, Control, and Dynamics, Vol. 20, No. 1, January-February 1997, pp. 74-80.

${ }^{19}$ Barron, A., Self-Organizing Methods in Modeling, chap. Predicted Squared Error: A Criteron for Automatic Model Selection, Marcel Dekker, 1984.

${ }^{20}$ Garza, F. and Morelli, E., "A Collection of Nonlinear Aircraft Simulations in MATLAB," Tech. Rep. TM-2003-212145, NASA, Hampton, VA, January 2003.

${ }^{21}$ Murch, A., "A flight control system architecture for the NASA AirSTAR flight test infrastructure," No. 2008-6990 in Guidance, Navigation, and Control Conference, AIAA, Honolulu, HI, August 2008.

${ }^{22}$ Wilborn, J. and Foster, J., "Defining Commercial Transport Loss-of-Control: A Quantitative Approach," No. 2004-4811 in Atmospheric Flight Mechanics conference, AIAA, Providence, RI, August 2004.

${ }^{23}$ Roskam, J., Airplane Flight Dynamics and Automatic Flight Controls, Roskam Aviation and Engineering Corporation, Lawrence, KS, 1979.

${ }^{24}$ Raymer, D., Aircraft Design: A Conceptual Approach, AIAA Education Series, AIAA, 3rd ed., 1999. 


\section{Tables}

Table 1. Aircraft simulation parameters

\begin{tabular}{ccccccccccc}
\hline \hline aircraft & description & $\begin{array}{c}\text { weight } \\
{[\mathrm{lbf}]}\end{array}$ & $\begin{array}{c}I_{x x} \\
{\left[\mathrm{slug} \cdot \mathrm{ft}^{2}\right]}\end{array}$ & $\begin{array}{c}I_{y y} \\
{\left[\mathrm{slug} \cdot \mathrm{ft}{ }^{2}\right]}\end{array}$ & $\begin{array}{c}I_{z z} \\
{\left[\mathrm{slug} \cdot \mathrm{ft}^{2}\right]}\end{array}$ & $\begin{array}{c}I_{x z} \\
{\left[\mathrm{slug} \cdot \mathrm{ft}^{2}\right]}\end{array}$ & $\begin{array}{c}S \\
{\left[\mathrm{ft}^{2}\right]}\end{array}$ & $\begin{array}{c}\bar{c} \\
{[\mathrm{ft}]}\end{array}$ & $\begin{array}{c}b \\
{[\mathrm{ft}]}\end{array}$ & $\begin{array}{c}x_{c g} \\
{[\mathrm{ft}]}\end{array}$ \\
\hline A-7 & fighter & 22699 & 16970 & 65430 & 76130 & 4030 & 375 & 10.8 & 38.7 & 0.30 \\
F-4 & fighter/bomber & 38924 & 24970 & 122190 & 139800 & 1175 & 530 & 16 & 38.67 & 0.29 \\
F-16C & fighter & 20500 & 9496 & 55814 & 63100 & 982 & 300 & 11.32 & 30 & 0.25 \\
F-16XL & fighter & 27867 & 18581 & 118803 & 135198 & 74 & 663 & 24.7 & 32.4 & 0.1 \\
F-106B & interceptor & 29776 & 18634 & 177858 & 191236 & 5539 & 698 & 23.75 & 38.13 & 0.25 \\
GTM & transport & 49.6 & 1.327 & 4.254 & 5.454 & 0.120 & 5.902 & 0.915 & 6.849 & 0.25 \\
TO & commuter & 10747 & 20922 & 24231 & 38425 & 1021 & 420 & 6.5 & 65 & 0.12 \\
X-31 & agility & 16000 & 3553 & 50645 & 49367 & 156 & 226.3 & 12.35 & 22.83 & 0.3 \\
\hline \hline
\end{tabular}

Table 2. Ranges and resolution for aerodynamic database interrogation

\begin{tabular}{cccccc}
\hline \hline case & variable & minimum & maximum & resolution & unit \\
\hline \multirow{4}{*}{ longitudinal } & $\alpha$ & -4 & +30 & 2 & $\mathrm{deg}$ \\
& $q$ & +0 & +50 & 5 & $\mathrm{deg} / \mathrm{s}$ \\
& $\delta_{e}$ & -20 & +10 & 2 & $\mathrm{deg}$ \\
\hline \multirow{5}{*}{ lateral/directional } & $\beta$ & +0 & +20 & 4 & $\mathrm{deg}$ \\
& $p$ & +0 & +100 & 20 & $\mathrm{deg} / \mathrm{s}$ \\
& $r$ & +0 & +50 & 10 & $\mathrm{deg} / \mathrm{s}$ \\
& $\delta_{a}$ & +0 & +10 & 2 & $\mathrm{deg}$ \\
\hline \hline
\end{tabular}

13 of 16 


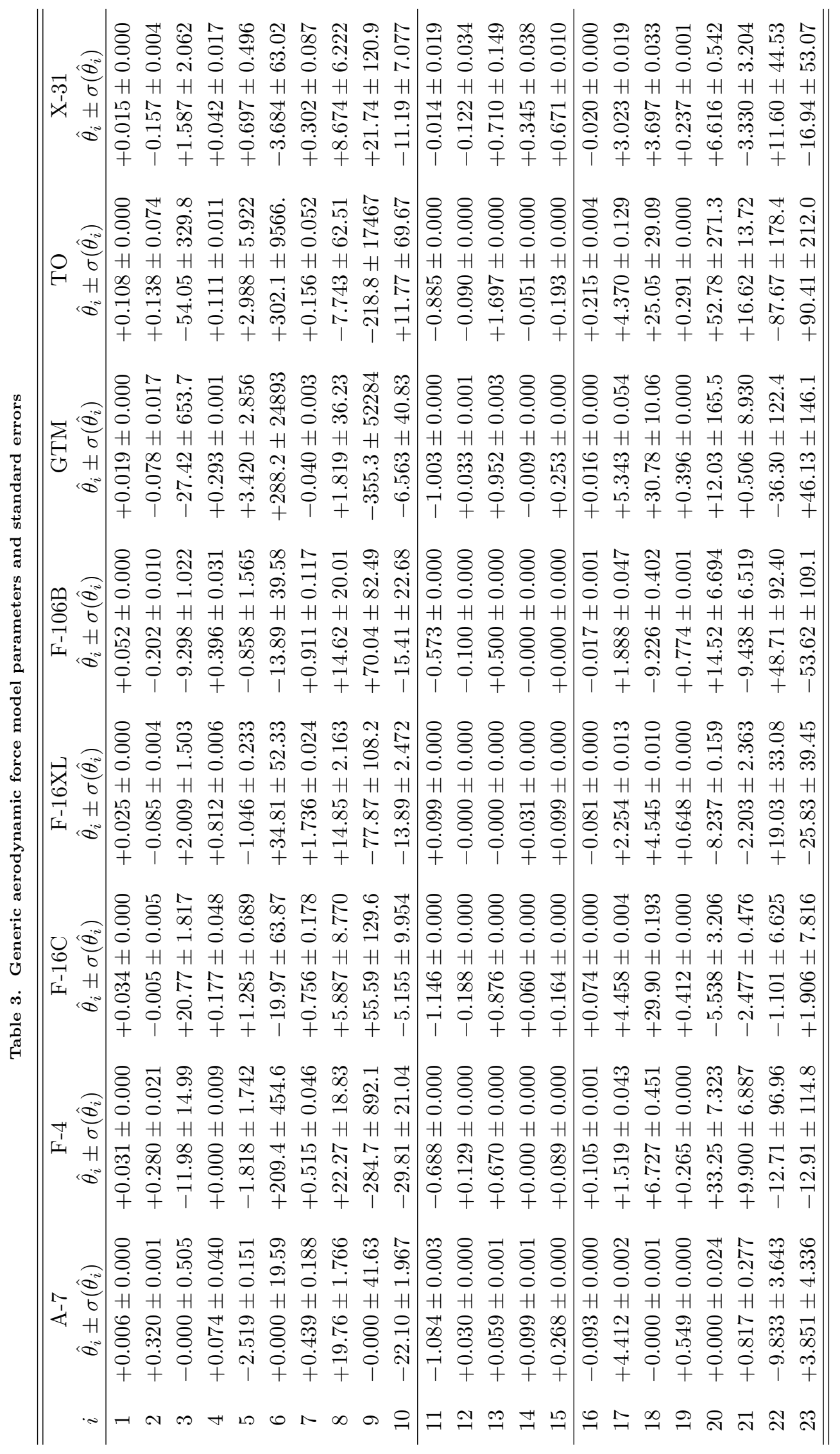




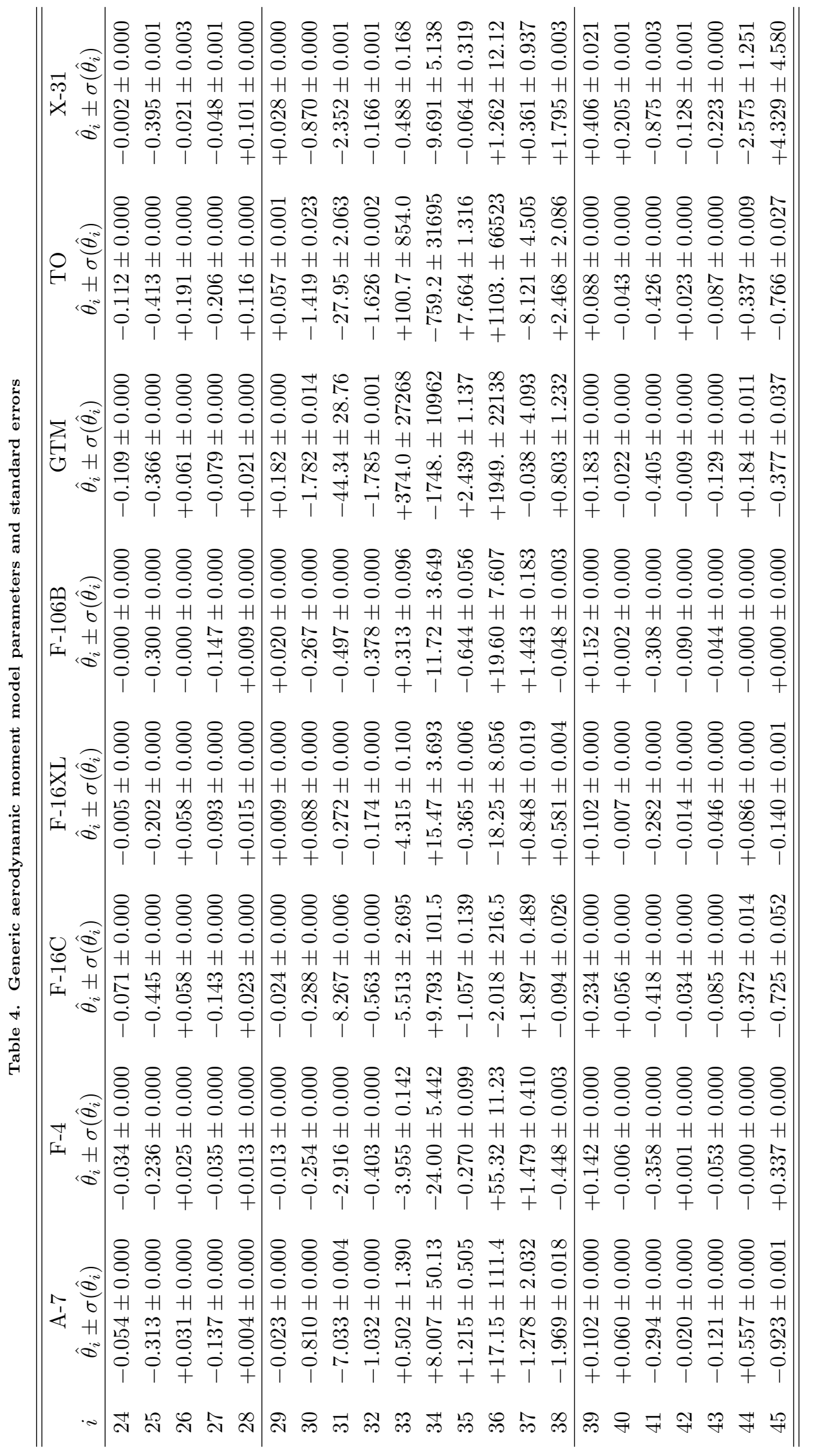


Table 5. Comparison of linearized Eigenvalues for the GTM

\begin{tabular}{ccccc}
\hline \hline Mode & \multicolumn{2}{c}{ Frequency $[\mathrm{rad} / \mathrm{s}]$} & \multicolumn{2}{c}{ Damping Ratio } \\
& Database & GNA & Database & GNA \\
\hline spiral & 0.0498 & 0.0887 & - & - \\
phugoid & 0.318 & 0.342 & 0.0517 & 0.0452 \\
roll subsidence & 5.28 & 5.44 & - & - \\
dutch roll & 5.89 & 5.27 & 0.149 & 0.179 \\
short period & 6.64 & 6.49 & 0.455 & 0.351 \\
\hline \hline
\end{tabular}

16 of 16 